\title{
ILCEA
}

Revue de l'Institut des langues et cultures

d'Europe, Amérique, Afrique, Asie et Australie

$12 \mid 2010$

La FASP : dix ans après...

\section{Territoire(s) culturel(s)}

Les Campus Novels de David Lodge

Sophie Gaberel-Payen

\section{(2) OpenEdition}

1 Journals

\section{Édition électronique}

URL : http://journals.openedition.org/ilcea/475

DOI : 10.4000/ilcea.475

ISSN : 2101-0609

Éditeur

UGA Éditions/Université Grenoble Alpes

Édition imprimée

ISBN : 978-2-84310-180-9

ISSN : 1639-6073

Référence électronique

Sophie Gaberel-Payen, « Territoire(s) culturel(s) », ILCEA [En ligne], 12 | 2010, mis en ligne le 29

septembre 2010, consulté le 20 avril 2019. URL : http://journals.openedition.org/ilcea/475; DOI :

10.4000/ilcea.475

Ce document a été généré automatiquement le 20 avril 2019

(C) ILCEA 


\title{
Territoire(s) culturel(s)
}

\author{
Les Campus Novels de David Lodge
}

\section{Sophie Gaberel-Payen}

\section{Introduction}

Nous nous proposons ici d'aborder la FASP universitaire de l'auteur professionnel et «professionnel-auteur » qu'est David Lodge : célèbre pour ses romans et ses ouvrages critiques, l'écrivain est en effet professeur émérite à l'université de Birmingham où il enseigna la littérature anglaise de 1960 à 1987, date à partir de laquelle il se consacra entièrement à l'écriture. Mêlant réalisme et métafiction, humour et sérieux, les facettes de cette écriture, à la fois culturellement ambitieuse et populaire, sont multiples - tout comme les reflets du miroir tendu à la réalité qu'il décrit, pastiche, parodie, met en scène.

1 Ainsi que le souligne Michel Petit, le « roman universitaire » (ou Campus Novel), bien que constituant un genre spécifique, traduit néanmoins parfaitement la notion de «fiction à substrat professionnel » (Petit, 2004, p. 19). Entreprise créative au sein de contraintes professionnelles, ce type de roman est écrit par des universitaires sur des universitaires et possède en effet les principales caractéristiques du genre (hormis celle du roman à suspense ou "thriller », en ce qui concerne les œuvres de David Lodge) : c'est une fiction où la représentation d'un milieu professionnel (de ses discours, de ses pratiques...) soustend l'intrigue, définit les personnages et intervient dans le dénouement.

2 L'angle privilégié pour aborder la FASP universitaire lodgienne sera ici celui de «la culture de spécialité ", c'est-à-dire, selon Françoise Raby, «l'ensemble des croyances, connaissances et des savoirs qui fait qu'un individu se reconnaît appartenir à une communauté ${ }^{1}$ ", une culture qu'elle définit par les notions de «lien» et de «clôture ». Notons d'ailleurs l'utilisation du mot latin campus (signifiant littéralement champ, domaine ), pour définir l'espace physique occupé par une université. Ce terme, qui apparut au début des années quarante aux États-Unis, fut ensuite utilisé en Angleterre dans les années cinquante. En revanche, il fut employé plus tardivement en France, car ne relevant pas vraiment d'une réalité culturelle française. 
3 Nous verrons comment David Lodge privilégie la notion de communication au sein de ses romans et comment ceux-ci offrent au lecteur en général, et à l'apprenant en langue et culture de spécialité en particulier, les clés permettant d'entrer dans ce domaine spécifique. Car, comme l'évoquait Umberto Eco dans sa préface du roman Un Tout petit monde (Small World, 1984) : "Si on lit des romans, c'est également pour acquérir les notions qui permettent de les lire. Ils nous introduisent à des mondes qui nous étaient inconnus et nous les rendent familiers. » (Lodge, 1991, p. 8)

4 Seront ainsi abordées non seulement la culture de l'ancrage géographique du récit fictionnel lodgien, notamment la ville de Rummidge (un Birmingham fictif cependant reconnaissable) mais aussi la culture d'une communauté spécialisée (universitaire) : en effet, héritier de Mary McCarthy (The Groves of Academe, 1952) ou encore Kingsley Amis ( Lucky Jim, 1954), David Lodge s'inscrit dans une tradition culturelle et littéraire qu'il souhaite perpétuer tout en la renouvelant. La conclusion d'Umberto Eco au sujet de Small World s'applique de fait tout autant à son auteur, à savoir qu'« il ne présuppose pas la connaissance d'une société : il la procure » (Lodge, 1991, p. 9).

\section{FASP universitaire et culture de spécialité}

La notion d'espace, de territoire, semble essentielle lorsqu'on aborde la fiction universitaire car inhérente à la définition de ce genre, notamment celle qu'en donne Christian Gutleben, c'est-à-dire : "Tout roman dont le monde universitaire constitue le cadre principal et dont les préoccupations universitaires constituent un thème essentiel. » (Gutleben, 1996, p. 6)

David Lodge rappelle d'ailleurs dans un essai intitulé Nabokov and the Campus Novel (Lodge, 2008b), que le terme latin campus, soulignant un espace référentiel restreint et clos, fut accolé à celui de novel dès lors que les universités américaines devinrent une source d'inspiration pour les écrivains. Quant à l'Angleterre, si dès le début du XIX ${ }^{e}$ siècle les deux grandes universités que sont Oxford et Cambridge apparurent dans des œuvres de fiction (soit comme cadre au sein duquel évoluait la jeunesse victorienne, soit comme objet de satire, et principalement dans le genre du Bildungsroman, c'est-à-dire du roman d'apprentissage), c'est après la Seconde Guerre mondiale que ce genre connut un réel essor. Conséquences des réformes du Butler Act de 1944, l'enseignement supérieur, jusquelà réservé à des classes aisées, fut en effet rendu plus accessible aux étudiants issus des classes moyennes et ouvrières - et les redbrick universities fleurirent dans le pays.

Lodge rappelle également que - à la différence de la France où la plupart des étudiants peuvent aller à l'université tout en continuant de vivre chez eux ou chez leurs parents le campus résidentiel, tel qu'il existe en Angleterre ou aux États-Unis, est un environnement très spécifique : c'est un lieu où la limite entre la vie sociale et la vie universitaire est clairement marquée et où l'on est sans doute plus à même de rencontrer des personnes dont les comportements sont la matière première de la fiction universitaire. Celle-ci décrit ainsi au lecteur un lieu, un microcosme au cœur duquel s'entremêlent des enjeux de puissance, de succès et de séduction et les sources de l'attraction qu'exercent les romans universitaires sont peut-être à trouver dans le fait qu'ils proposent la représentation d'un milieu où les principes, les objectifs et les conflits qui gouvernent toute vie communautaire sont représentés. 
Quant à l'écrivain, c'est une trilogie (la célèbre trilogie de Rummidge) qu'il proposa au lecteur dans les années soixante-dix et quatre-vingt, avec d'abord Changement de décor ( Changing Places, 1975), où deux professeurs d'université, l'anglais Phillip Swallow et l'américain Morris Zapp, échangent leurs postes... puis leurs femmes ; Un Tout petit monde (Small World, 1984), « romance académique » où ces derniers croisent le chemin du jeune Persse McGarrigle allant de conférence en conférence à travers le monde, à la poursuite de la belle Angelica Pabst dont il est amoureux ; et Jeu de société (Nice Work, 1988) dont les personnages (Vic Wilcox, directeur général d'usine et Robyn Penrose, maître de conférences, spécialiste du roman industriel $d u$ xIX ${ }^{e}$ siècle) rejouent les intrigues du roman victorien.

Ces histoires se situent dans un milieu professionnel possédant ses propres codes, ses coutumes, ses rituels, des savoirs et des savoir-faire, un milieu peuplé de personnages dont les préoccupations, les conflits et les petites lâchetés sont traités de façon souvent très divertissante. Ce sont ces rituels, ces codes, en un mot, une culture, qui sont décrits au lecteur par l'intermédiaire de scènes récurrentes décrivant des colloques ou encore des congrès, comme c'est le cas dans le prologue d'Un Tout petit monde:

Le congrès de l'âge moderne ressemble au pèlerinage des chrétiens du Moyen Âge dans la mesure où il permet à ses participants de se livrer à tous les plaisirs et à tous les divertissements inhérents au voyage sans qu'ils aient à se départir de cet air austère qui sied à toute recherche pour se mieux connaître. Reste bien sûr que certains exercices pénitentiels doivent être accomplis : faire une communication, peut-être, et naturellement écouter celle des autres. Mais grâce à cet alibi, vous visitez des lieux nouveaux et intéressants, vous rencontrez des gens nouveaux et intéressants et créez avec eux des relations nouvelles et intéressantes. (p. 17)

Dans Jeu de société, ce sont des cours (véritable représentation du discours professionnel en action) qui sont décrits, comme le cours magistral que donne Robyn sur le roman industriel du XIXe siècle; tandis qu'elle souligne comment, dans les ouvrages de cette période (notamment ceux écrits par des femmes), les contradictions idéologiques envers la révolution industrielle prennent souvent une connotation sexuelle, les réactions des étudiants sont dépeintes avec une pointe d'humour :

Lorsqu'elle prononce le mot « sexuelle », un frémissement d'intérêt court parmi ses auditeurs toujours silencieux. Ceux qui rêvassaient ou gravaient leurs initiales sur les pupitres redressent la tête. Ceux qui prenaient des notes continuent de le faire avec encore plus d'application. On cesse de tousser, de renifler, de remuer les pieds. Tandis que Robyn poursuit, le son de sa voix n'est troublé périodiquement que par le froissement d'une feuille 21 x 29,7 que l'on arrache brusquement d'un bloc-notes. (p. 84)

Ce sont aussi des traditions parfois très protocolaires qui sont présentées au lecteur à travers des passages expliquant le fonctionnement du monde universitaire britannique, comme dans La Vie en sourdine (Deaf Sentence, 2008a) qui relate l'histoire de Desmond Bates, professeur de linguistique retraité souffrant de surdité et menant une vie sans surprise jusqu'à ce qu'il rencontre Alex Loom, une étrange doctorante américaine. Desmond repense, parfois avec nostalgie, au calendrier universitaire :

Il se mit à regretter [...] l'arrivée des nouveaux étudiants excités et pleins d'espoir chaque automne; la soirée de Noël du département avec ses traditionnels sketchs d'étudiants imitant les tics et le jargon favori des membres du personnel; la semaine de séminaire pendant le deuxième trimestre où l'on emmenait les deuxième année passer quelques jours dans un centre du District des Lacs; les réunions d'examinateurs pendant le troisième trimestre au cours desquelles, tous assis autour d'une longue table encombrée de devoirs déjà notés et de dissertations touffues, ils calculaient et classaient les résultats définitifs comme des dieux 
dispensant des récompenses et des punitions à des mortels ; et finalement la remise des diplômes avec cortège et accompagnement à l'orgue dans le grand amphi où l'on écoutait l'orateur de l'université résumer ad nauseam les performances des licenciés ayant reçu des mentions, serrant ensuite la main de parents très fiers et de leurs progénitures en toge, sirotant un punch aux fruits sous la grande tente plantée sur la pelouse circulaire, après quoi tout le monde se dispersait et allait prendre de grandes vacances bien méritées. (p. 44-45)

Le fait que des auteurs issus d'un milieu professionnel prennent ce dernier pour objet de fiction, offrant ainsi une représentation de la culture collective de ce milieu, est intéressant à bien des égards. D'abord parce que les rôles de professeur et de romancier se mélangent souvent, le premier analysant lui-même son œuvre fictionnelle, le second écrivant de la fiction tout en continuant à enseigner la littérature et les théories littéraires. La FASP universitaire, écrite dans un style souvent auto référentiel, est ainsi l'expression d'une attitude critique envers son propre mode de fonctionnement, une fiction qui pourrait être décrite à la fois comme "miroir et lampe ", pour reprendre les termes de A.H.Abrams (1953), c'est-à-dire informant le lecteur tout en jetant un éclairage qui dévoile les relations existant entre le roman et une tradition littéraire. Maurice Couturier, traducteur et ami de Lodge, a quant à lui tenté d'expliquer le succès de l'écrivain, notamment en France :

La culture universitaire s'est beaucoup transformée au cours des vingt dernières années, devenant internationale, avec des enseignants voyageant à travers le monde [...] Il me semble que de nombreuses personnes, n'étant pas universitaires, ont trouvé intéressant de découvrir comment ce monde fonctionne. [Small World] est d'une certaine façon une encyclopédie de critique littéraire, non seulement de ce siècle mais remontant également jusqu'au XVIII ${ }^{\mathrm{e}}$ siècle ou même jusqu'à la Renaissance. Voici quelques raisons [...] pour lesquelles les romans de David ont autant de succès $[\ldots]^{2}$

Dans les années quatre-vingt, Lodge conquit en effet, en France comme ailleurs, un lectorat assez considérable. Tenter de le conserver, sans jamais transiger sur ses ambitions littéraires, est très important pour lui et sa popularité vient sans nul doute du fait qu'il ravit à la fois le lecteur « innocent », qui se délecte de voir la vie universitaire si bien décrite et le lecteur "actif " qu'il entraîne dans une incessante quête de sens, de références intertextuelles, écrivant des romans qui, comme il le dit lui-même, sont faits pour être lus plus d'une fois, « un genre de fiction composée de différentes strates, afin qu'elles fassent sens et donnent satisfaction même en surface, tandis qu'il existe d'autres niveaux de compréhension et de référence, prêts à être découverts par ceux qui ont l'intérêt ou la motivation pour le faire " (Haffenden, 1985, p. 160).

Ainsi qu'il nous le confiait dans un entretien ${ }^{3}$, Lodge accepte tout à fait d'être défini comme un auteur de fiction universitaire tout en refusant de n'être que cela, revendiquant l'équilibre entre tradition et innovation dans ses ouvrages :

Le terme Campus Novel est quelque peu stéréotypé et c'est pourquoi certains romanciers écrivant sur le milieu universitaire (comme mon ami le défunt Malcolm Bradbdury) n'apprécient pas qu'il soit appliqué à leur œuvre. Je n'y vois pas d'objection même si j'ai toujours cherché à expérimenter, à dépasser la forme du roman universitaire classique [...] qui est habituellement centré sur une université et ses occupants; j'ai également essayé de traiter divers aspects de la vie universitaire et de l'utiliser comme un microcosme où s'illustreraient des thèmes plus vastes. Dans Changement de décor il y a deux campus, dans Un Tout petit monde il y a un campus international et dans Jeu de société, l'université est opposée au monde $\mathrm{du}$ commerce et de l'industrie. Et ces trois romans traitaient respectivement de la contre-culture des années soixante, de la mondialisation d'une culture savante dans 
les années soixante-dix et de l'impact de la révolution économique thatchérienne sur l'Angleterre des années quatre-vingt. [Notre traduction.]

Si les Campus Novels représentent « un étroit petit monde » pour reprendre les termes qu'utilisait Bakhtine (1978, p.90), avant Lodge, ces romans entrent cependant en interaction avec d'autres petits mondes et peuvent être considérés comme de véritables documents gardant les traces de l'évolution d'une société.

La FASP universitaire semble ainsi se jouer des frontières et posséder un statut intermédiaire par rapport à la FASP traditionnelle. En premier lieu, parce que la caractéristique du roman à suspense ou thriller n'est pas toujours associée à ce genre bien que ce soit parfois le cas, comme dans Death in a Tenured Position(1981). Dans ce roman d'Amanda Cross (nom de plume de Carolyn Heilbrun), le corps d'une professeure nouvellement en poste est retrouvé inerte dans les toilettes pour hommes et l'intrigue consiste, entre autres, à découvrir qui l'a empoisonnée. Ensuite parce que cette fiction, bien que décrivant le plus souvent la vie d'enseignants en Lettres et Sciences humaines (car c'est là que travaillent la plupart des auteurs-enseignants), aborde de plus en plus d'autres domaines tels que les sciences, comme Pensées secrètes (Thinks..., 2001) de Lodge lui-même - roman qui narre la confrontation, la séduction et enfin la brève liaison amoureuse entre Ralph Messenger (chercheur spécialiste des sciences cognitives) et Helen Reed (romancière).

La FASP universitaire est donc une FASP « interstitielle », pour reprendre les termes de Shaeda Isani (Petit, 2004, p. 31-32), ne se situant pas seulement « aux limites » du genre mais plutôt, nous semble-t-il, à une intersection entre plusieurs genres, proposant au lecteur un véritable palimpseste générique. Jeu de société, par exemple, oppose puis entremêle deux idéologies politiques, deux cultures à travers les personnages de Vic Wilcox et de Robyn Penrose. C'est ainsi qu'après avoir fait découvrir le monde de l'industrie à une Robyn médusée, Vic, non moins surpris, découvre le milieu universitaire et apprend de la bouche de la jeune femme qu' « ici [...] la lecture est un travail. La lecture est une production. Et ce que nous produisons, c'est du sens » (p. 358).

Mais ce roman oppose également deux styles littéraires (la métafiction et le réalisme) et propose une réécriture postmoderne des romans victoriens, des Condition-of-England Novels, ceux écrits par George Eliot, Charlotte Brontë, Charles Dickens, etc. Il est d'ailleurs intéressant de noter que l'un des titres auquel avait d'abord pensé Lodge pour Jeu de société (Nice Work) était Hard Times in Rummidge, en hommage à l'écrivain... Quant à l'histoire de Robyn, elle se confond notamment avec celle de l'héroïne d'Elizabeth Gaskell dans North and South (1854-1855).

11 Tout le talent de Lodge réside donc dans la façon dont il jette des passerelles entre ces différents mondes/genres au sein de son univers fictionnel; peut-être, comme il le rappelle dans The Art of Fiction, parce que le roman, de part sa nature même " incorpore différents styles, ou voix, qui, pourrait-on dire, communiquent les unes avec les autres ainsi qu'avec d'autres voix en dehors du texte c'est-à-dire le discours de la culture et de la société au sens large » (Lodge, 1992, p. 128) [Notre traduction].

\section{Une FASP à la fois populaire et culturellement ambitieuse}

L'écriture de Lodge est à la fois culturellement ambitieuse et populaire : son humour, s'illustrant souvent au travers d'un comique de situation, explique son succès auprès d'un 
lectorat non-universitaire. Exposer, railler les comportements de la profession, fait partie des caractéristiques de la FASP universitaire. Comme le note Christian Gutleben, « dès l'instant où le genre se tourne vers un large public pour dénoncer les excentricités de l'élite enseignante, il suscite un [...] plaisir populaire, celui du détrônement, de la désacralisation » (Gutleben, 1996, p. 153).

Au demeurant, le lien entre humour textuel et sexualité, présent dans chaque œuvre de David Lodge, est souvent fortement lié à l'identité même des personnages de ses romans, soit comme synonyme d'inspiration créatrice, soit comme substitut de la gratification pouvant découler de leur travail. Il participe pour le lecteur d'un même principe de découverte, de plaisir, voire de jouissance, ainsi que l'évoquait Roland Barthes, reliant ces sensations à celles procurées à la fois par l'acte sexuel et par l'acte de lecture. Morris Zapp, dans Un Tout petit monde, n'avait-il pas intitulé l'une de ses communications universitaires "Textualité et strip-tease"? Dans La Vie en sourdine, il est vrai, les personnages de Lodge ont vieilli et l'adultère semble avoir laissé la place à une certaine harmonie conjugale. Si le sexe était une sublimation de l'instinct de travail pour Morris Zapp, près de vingt ans plus tard, il est devenu un objet d'anxieuse anticipation pour Desmond.

13 Quant au comique culturel, il crée une communauté de lien avec un public plus littéraire. Lucie Olbrechts-Tyreca souligne en effet l'importance du «rire qui naît dans la connivence, la communication [...]. Le rire est ainsi une manière d'attester que l'on sait ce qu'il faut savoir pour faire partie du groupe » (Olbrechts-Tyreca, 1974, p. 396-397). La notion de plaisir ludique est ainsi inhérente à l'œuvre de Lodge qui définit d'ailleurs le roman comme un jeu nécessitant au moins deux joueurs, un écrivain mais également un lecteur (Lodge, 1988, p. 74). À ce dernier en effet de reconnaître les références intertextuelles, ces relations entre hypotexte et hypertexte qui créent un réseau d'interactions représentant le fondement, le socle d'une culture donnée.

Ces références intertextuelles peuvent parfois être « incestueuses » (Gutleben, 1996) c'està-dire faire référence à d'autres fictions universitaires. Les styles de Lodge et de Malcolm Bradbury (ayant lui-même écrit des romans universitaires dès 1959), par exemple, ont été parfois confondus par certains lecteurs, et ont souvent été comparés et rapprochés par les critiques mais également par d'autres écrivains. Dans Coming From Behind, de Howard Jacobson, le protagoniste nomme «Bradbury Lodge » une hypothétique maison où se rencontreraient célébrités littéraires et universitaires; dans Floating Down to Camelot, de David Benedictus, un étudiant regrette de ne pas s'être inscrit dans une université d'East Anglia car «Bradbury y est et Lodge est à Birmingham » (p. 38).

Les deux écrivains se sont eux-mêmes très souvent amusés dans leurs romans à faire référence à leurs œuvres respectives et ont joué de cet amalgame. Dans Rates of Exchange, Bradbury fait par exemple allusion à un écrivain, Brodge, qui aurait écrit Changing Westward (mélange de Changing Places et de Stepping Westward). Dans My Strange Quest for Mensonge: Structuralism's Hidden Hero, la postface aurait été écrite par Michel Tardieu (Professeur de Narratologie Structuraliste à l'université de Paris, qui n'est autre qu'un personnage d'Un Tout petit monde), et traduite par... David Lodge. Quant à ce dernier, il insère un passage dans Un Tout petit monde, où deux romanciers anonymes, mais ressemblant étrangement aux deux écrivains, sont en pleine conversation. Enfin, dans $L a$ Vie en sourdine, Desmond remarque que cela ne l'étonnerait pas d'apparaître un jour, sous des traits à peine déguisés, dans un roman universitaire. (p. 403) 
Les références intertextuelles peuvent également être "restreintes " (Ricardou, 1976, p. 282) c'est-à-dire souligner les rapports textuels entre les textes d'un même auteur. Lorsque Helen, dans Pensées secrètes, essaie de choisir un passage à analyser pour sa communication à l'Institut de sciences cognitives, elle pense d'abord étudier une scène tirée des Ambassadeurs de Henry James mais rejette ensuite cette idée car « cela a déjà été fait »; Lodge expliquera dans un essai intitulé « A Conversation about Thinks... » que cette plaisanterie auto référentielle repose sur le fait que lui-même avait fait cette analyse dans Language of Fiction (Lodge, 2002, p. 296-297). Toujours dans Pensées secrètes, c'est aussi Robyn Penrose, la protagoniste de Jeu de société, qui apparaît brièvement pour participer à une conférence; la jeune femme est devenue responsable du département de la communication et de la culture à l'université de Walsall (p. 226).

17 L'interaction entre deux mondes, celui où l'on raconte (extradiégétique, réel) et celui que l'on raconte (intradiégétique, fictionnel) a d'ailleurs été soulignée par le Professeur américain Elaine Showalter dans une étude critique sur le roman universitaire intitulée Faculty Towers: the Academic Novel and its Discontents (2005) ; elle affirme en effet avoir découvert quelques personnages de romans lui ressemblant au cours de ses recherches. Ici encore, on le voit, les frontières entre différents territoires, notamment entre réalité et fiction, sont mouvantes.

Si la FASP permet à un lectorat non-universitaire de découvrir un milieu spécialisé, elle représente également une aide didactique fort intéressante pour un public plus ciblé. Avec l'apprenant en «langue de spécialité », étudiant spécialiste d'anglais (en Langues, Littératures et Civilisations Étrangères par exemple), la FASP universitaire peut être utilisée pour aborder un genre littéraire, un lexique spécialisé, notamment en méthodologie de la critique littéraire. Elle peut ainsi être un outil pédagogique, un support d'enseignement très utile, offrant les clés qui permettront à l'apprenant d'acquérir une compétence langagière mais aussi de la contextualiser. Les termes langue et culture sont en effet dans un rapport dialogique indissociable et c'est d'ailleurs ce que rappelle Desmond à ses étudiants, dans La Vie en sourdine, lors de son cours d'introduction :

La langue est ce qui fait que nous sommes humains, ce qui nous distingue d'une part des animaux et d'autre part des machines, ce qui fait de nous des êtres conscients, capable de pratiquer les arts, les sciences, tout ce qui fait la civilisation. C'est la clé [...] Pour comprendre la culture et la société, il faut être capable d'en analyser le discours. (p. 46-47)

Certaines expressions ou bien certains termes utilisés en analyse littéraire possèdent une " charge culturelle partagée " (Gallisson, 1987) à la fois en français et en anglais : les termes metaphor et metonimy sont ainsi reconnaissables par des étudiants mais la FASP peut aider à mieux les comprendre en alliant divertissement et apprentissage (on se rappellera à ce sujet le passage à la fois humoristique et didactique où Robyn revient sur cette différence dans Jeu de société). Elle peut aussi illustrer certaines définitions phonologiques; par exemple lorsque Desmond explique ses difficultés à différencier certaines consonnes à cause de sa surdité et définit la consonne [f] comme étant à la fois une fricative labiodentale et une continue, ainsi appelée :

[...] parce que pour produire ce phénomène il faut mettre vos dents supérieures en contact avec votre lèvre inférieure et laisser de l'air s'échapper entre les deux. On dit aussi que c'est une continue parce que vous pouvez continuer à faire le son aussi longtemps que votre souffle le permet: ffffffffffffff..., bien que je me demande pourquoi vous voudriez le faire, sauf peut-être si vous commencez à dire « Fuck» et changez d'avis. (p. 31) 


\section{Culture d'un ancrage géographique : « Rummidge »}

Enfin, la culture de l'ancrage géographique du récit fictionnel lodgien est déterminante. Une ville du nord de l'Angleterre est en effet devenue indissociable de l'œuvre de David Lodge dans la mémoire collective : Rummidge. Cette ville (pendant fictif de Birmingham, ainsi que cela a été rappelé supra, où Lodge enseigna et où il vit toujours) est ainsi devenue un véritable référent géographique, celui d'une tradition et d'un espace textuels. Elle fut rendue célèbre par la trilogie de Rummidge et notamment par la note d'auteur figurant en exergue de Jeu de société :

Il faut peut-être que j'explique, pour les lecteurs qui ne sont jamais venus ici auparavant, que Rummidge est une ville imaginaire, avec des universités imaginaires et des usines imaginaires, habitée par des gens imaginaires, et qui occupe, pour les besoins de la fiction, l'endroit où se situe Birmingham sur les cartes du monde prétendu réel. (p. 9)

Lodge s'est souvent exprimé sur cette frontière incertaine, devenue de plus en plus transparente avec le temps, entre les deux villes; le fait que Rummidge soit une (re)construction fictionnelle de Birmingham faisant d'ailleurs partie des conventions aisément acceptées par les lecteurs. L'interpénétration entre deux mondes, l'un réel et l'autre fictionnel, s'est par ailleurs trouvée renforcée lors de l'adaptation télévisée de ce roman produit par Chris Parr en 1989 pour la BBC (et dont Lodge écrivit le scénario) : le tournage se déroula en effet exclusivement à Birmingham, notamment au sein de l'Université où divers lieux servirent de décor aux scènes d'extérieur (le campus) et d'intérieur (dont le « General Office » en lieu et place des bureaux de Robyn Penrose et de Phillip Swallow).

Reprenant les termes d'Oscar Wilde, l'écrivain rappelle par ailleurs dans « Nabokov and the Campus Novel» (Lodge, 2008b), que non seulement l'art imite la vie mais qu'il existe parfois le risque que la vie imite l'art:

J'ai moi-même par deux fois inventé des universités qui, quelques années plus tard,

à ma grande confusion, ont été construites dans le monde réel : University College à

Limerick (inspirée par Un Tout petit monde)et l'université de Gloucester (inspirée par

Pensées secrètes). [Notre traduction.]

En ce qui concerne Rummidge et Birmingham, elles sont liées l'une à l'autre à un tel point que l'écrivain qui avait d'abord songé situer son dernier roman, La Vie en sourdine, à Rummidge, lui a préféré une autre ville du nord de l'Angleterre qui n'est, elle, jamais nommée - ceci afin d'éviter que le roman soit considéré par le lecteur comme étant entièrement autobiographique.

Il est enfin intéressant de noter que ce roman a été qualifié de " post-universitaire » par certains critiques (Lichtig, 2008), puisque le protagoniste est cette fois un professeur à la retraite. Le préfixe "post » gêne cependant quelque peu l'écrivain puisque selon lui, il s'appliquerait davantage à une notion liée au temps, qu'à une notion liée à un lieu.

\section{Conclusion}

Pour conclure, il semble que la FASP universitaire, de par sa nature hybride, offre au lecteur la possibilité de découvrir non seulement un milieu spécialisé mais également divers territoires culturels, qu'ils soient littéraires, didactiques, géographiques, réels et/ ou fictionnels. 
Le jeu entre réalité et fiction est ainsi à la source de toute FASP. David Lodge souligne d'ailleurs à ce propos qu' « un écrivain qui travaille dans un environnement institutionnel dans lequel il trouve la matière de ses romans et dont il s'inspire, est particulièrement sensible à l'interaction entre le réel et la fiction, traversant encore et encore la frontière entre ces deux mondes.» (Lodge 2008b) Bien qu'il ait lui-même rapidement quitté le milieu du professorat pour vivre de sa plume, attentif à marquer une certaine distinction, quoiqu'artificielle, entre sa vie d'enseignant et sa vie de romancier, il est toujours resté au contact du monde universitaire, et est souvent plébiscité pour son œuvre. La notion d'interaction, comme celle de palimpseste générique, est donc incontournable: elle donne même toute sa saveur à la FASP universitaire en général et aux Campus Novels de David Lodge en particulier.

\section{BIBLIOGRAPHIE}

\section{Romans}

BENEDICTUS, David, Floating Down to Camelot, Londres, Macdonald, 1985.

BRADBURY, Malcolm, Stepping Westward, Londres, Secker, 1965.

-, My Strange Quest for Mensonge..., Deutsch, 1987.

LODGE, David, Jeu de société (Nice Work, 1988), Paris, Payot \& Rivages, 1990.

-, Changement de décor (Changing Places, 1975), Paris, Payot \& Rivages, 1991.

-, Un Tout petit monde (Small World, 1984), Paris, Payot \& Rivages, 1991.

-, Pensées secrètes (Thinks..., 2001), Paris, Payot \& Rivages, 2004.

-, La Vie en sourdine (Deaf Sentence, 2008), Paris, Payot \& Rivages, 2008 a.

\section{Ouvrages critiques}

ABRAms, M. H., The Mirror and the Lamp, Oxford, OUP, 1953.

BAKHTINE, Mikhail, Esthétique et théorie du roman, Paris, Gallimard, 1978.

GABEREL-PAYEN, Sophie, « Deafinitely Lodgean. An Interview with David Lodge », Ebc 38, Presses universitaires de la Méditerranée, 2010.

GALLISSON, Robert, Accéder à la culture partagée par l'entremise des mots à C.C.P., ELA, n 67, Paris, Didier Erudition, 1987.

GUTLEBEN, Christian, Un Tout petit monde : le roman universitaire anglais - 1954-1994, Presses universitaires de Strasbourg, 1996.

HAFFENDEN, John, « David Lodge », Novelists in Interview, London, Methuen, 1985.

ISANI, Shaeda, "The FASP and the Genres within the Genre ", dans Aspects de la fiction à substrat professionnel : du général au particulier, Michel Petit (dir.), coll. « Travaux 20.25 », Presses universitaires de Bordeaux, 2004. 
LODGE, David, Write On, Occasional Essais. 1965-1985, [1986], Londres, Harmondsworth, Penguin, 1988.

-, The Art of Fiction, London, Secker and Warburg, 1992.

-, Consciousness and the Novel: Connected Essais, New York, Harvard University Press, 2002.

OlBREChtS-TYRECA Lucie, Le Comique du discours, Presses universitaires de Bruxelles, 1974.

PETIT Michel, « La fiction à substrat professionnel : une autre voie d'accès à l'anglais de spécialité ». Aspects de la fiction à substrat professionnel : du général au particulier. Michel Petit (dir.), coll. « Travaux 20.25 », Presses universitaires de Bordeaux, 2004.

RICARDOU Jean, dans Lucien Dällenbach, « Intertexte et autotexte », Poétique, 1976.

SHOWALTER Elaine, Faculty Towers: the Academic Novel and its Discontents, Oxford University Press, 2005.

Sites web

LICHTIG Toby, «Is Silence Really Golden », The Observer, 11 mai 2008.

LODGE David, « Nabokov and the Campus Novel », Cycnos, vol. 24, $\mathrm{n}^{\circ}$ 1, 2008b, [En ligne], consulté le 20 mars 2009.

RABY Françoise, « Les relations entre savoirs linguistiques et savoirs de spécialité dans la construction des processus de passage de l'être "cultivé" à l'être "civilisé" ", [En ligne], consulté le 04 mai 2009.

\section{NOTES}

1. F. Raby, «Les relations entre savoirs linguistiques et savoirs de spécialité dans la construction des processus de passage de l'être "cultivé" à l'être "civilisé" ", [en ligne].

2. M. Couturier, Enregistrement du colloque intitulé "L'Auteur et son Traducteur ", organisé à l'université de la Sorbonne, le 13 mars 1999 par le centre de recherche ERCLA, Paris IV.

3. S. Gaberel-Payen, "Deafinitely Lodgean. An Interview with David Lodge", Ebc 38, 2010, p. 91-100.

\section{RÉSUMÉS}

Cet article se propose d'aborder la FASP universitaire de l'écrivain David Lodge et de souligner comment le Campus Novel traduit la notion de "fiction à substrat professionnel ", bien qu'il constitue un sous-genre spécifique. De par sa nature hybride, cette fiction, à la fois populaire et culturellement ambitieuse, offre aux lecteurs non seulement la possibilité de découvrir un milieu spécialisé mais également les clés permettant d'accéder à divers territoires culturels, qu'ils soient littéraires, didactiques, géographiques, réels et/ou fictionnels.

Though often considered a somewhat marginal sub-genre of the FASP, this paper seeks to demonstrate the essential FASP characteristics of the campus novel through an analysis of David Lodge's novels. This naturally hybrid form of fiction - both popular and culturally ambitious - 
enables readers to discover a specialised environment and its various cultural territories, whether literary, didactic, geographical, real and/or imaginary.

INDEX

Mots-clés : FASP universitaire, roman universitaire, David Lodge, culture de spécialité, territoires réels et fictionnels, hybridité

Keywords : Academic FASP, Campus Novel, David Lodge, specialised culture, real and imaginary territories, hybridity

\section{AUTEUR}

\section{SOPHIE GABEREL-PAYEN}

Université d'Evry 